Ciência e Natura, Santa Maria, v. 37 n. 4 set-dez. 2015, p. 171-185

Revista do Centro de Ciências Naturais e Exatas - UFSM

ISSN impressa: 0100-8307 ISSN on-line: 2179-460X

\title{
Aplicação de Sensoriamento Remoto na Reconstrução da Série Temporal da Temperatura Mesosférica para uma Região com Baixa Latitude
}

\author{
Application of Remote Sensing in the Reconstruction of Temporal Series of mesospheric temperature for a \\ Region with Low Latitude
}

Priscila Valdênia dos Santos ${ }^{1}$, Ricardo Arlen Buriti ${ }^{2}$

${ }^{1}$ Departamento de Física Teórica e Experimental, Universidade Federal do Rio Grande do Norte ${ }^{2}$ Unidade Acadêmica de Física, Universidade Federal de Campina Grande

\begin{abstract}
RESUMO
Medidas da temperatura atmosférica para altitudes entre 80 e $100 \mathrm{~km}$ a partir do coeficiente de difusão ambipolar observado por radar meteórico é uma técnica relativamente nova. Porém, devido à falta de informações menos incertas dos modelos de pressão atmosférica nessa região, esta técnica apresenta problemas. Surgiu, então, a necessidade de se desenvolver uma técnica na qual a temperatura, na altitude onde ocorre a maior contagem de meteoros, é calculada a partir do gradiente de temperatura (Hocking, 1999). Neste trabalho aplicamos tal técnica e a temperatura foi calculada para cada dia de observação entre julho de 2004 e junho de 2009. Os valores foram comparados com dados de temperatura do TIMED/ SABER e com dados de temperatura rotacional do OH(6,2) obtida pelo fotômetro Multi-3, no sítio de observação de São João do Cariri-PB $\left(7,4^{\circ} \mathrm{S} ; 36,5^{\circ} \mathrm{W}\right)$. A média diária anual da temperatura, obtida pelos equipamentos, apresentou forte variação sazonal com máximos nos meses em torno dos equinócios, porém, com valores médios bem distintos. A média total obtida pelo radar meteórico SKiYMET foi de 226,2 K, acima das médias dos outros instrumentos. As temperaturas mesosféricas foram submetidas à análise espectral e os resultados revelaram a presença de oscilações de 80, 181,7, 274,5, 364 e 718 dias, para os dados do radar meteórico SKiYMET. Para a temperatura rotacional, as oscilações observadas foram 183,1, 403,9 e 647 dias. Nos dados do TIMED/SABER, as oscilações detectadas nas 3 altitudes analisadas foram de 90 , $\sim 182,5$ e 365 dias.
\end{abstract}

Palavras-chave: Temperatura Mesosférica, Sensoriamento Remoto, Baixas Latitudes

\section{A B S TRACT}

Atmospheric temperatures measurements for the height between 80 and $100 \mathrm{~km}$ from the ambipolar diffusion coefficient taken by meteor radar is a relatively new technique. However, due to lack of more accurate models of atmospheric pressure in the region, this technique has problems. Then it became necessary the development of a technique in which the temperature in the altitude where the highest count of meteors occurs is calculated from the temperature gradient. In this work the temperature was calculated for each day of observation between July 2004 and June 2009. The values were compared with temperature data from TIMED/SABER and $\mathrm{OH}(6,2)$ rotational temperature data obtained by multichannel photometer at the site of observation at São João do Cariri-PB $\left(7,4^{\circ} \mathrm{S} ; 36,5^{\circ} \mathrm{W}\right)$. The daily average annual temperature obtained by each equipment showed strong seasonal variation with maxima at the months near of the equinoxes, however, with averaged values very different. The average total obtained by the meteor radar SKiYMET was $226.2 \mathrm{~K}$, higher than the average of other instruments. Mesospheric temperatures were subjected to spectral analysis and the results revealed the presence of oscillations of 80,181.7, 274.5, 364 and 718 days in the meteor radar SKiYMET. For the rotational temperature, the observed oscillations were 183.1, 403.9 and 647 days. In the TIMED/SABER data, the oscillations detected in the three different analyzed altitudes were $\sim 90, \sim 182.5$ and $\sim 365$ days. These results were compared with those obtained elsewhere and reported in the literature.

Keywords: Mesospheric Temperature, Remote Sensing, Low Latitudes 


\section{Introdução}

No estudo da atmosfera terrestre, a região entre 20 e $100 \mathrm{~km}$ de altura, também denominada de média atmosfera, tem recebido grande atenção devido à ênfase que vem sendo dada às mudanças globais. Esta região contém a maior parte do ozônio da atmosfera da Terra

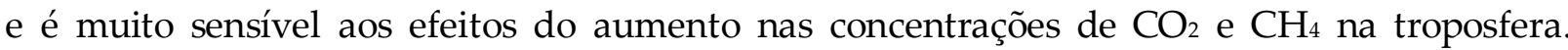
Alguns estudos têm mostrado que, dobrando-se essas concentrações, ocorre uma diminuição de 10 a $20^{\circ} \mathrm{C}$ na temperatura da média atmosfera. A média atmosfera apresenta uma série de características importantes e interessantes, em que o conhecimento da temperatura em função da altura exerce um papel crucial para a dinâmica nesta faixa de altitude. Dessa forma, para compreender a física e a química dessa região é necessário conhecer o seu perfil de temperatura.

Vários processos dinâmicos ocorrem na média atmosfera terrestre e a presença de oscilações de grandes amplitudes e de longos períodos é uma das características da circulação zonal média dessa região (ALVES, 2007). Enquanto o escoamento zonal médio na região apresenta uma oscilação anual em médias e altas latitudes, no Equador e na região de baixas latitudes os ventos são caracterizados por uma oscilação quase bi-anual (Quase-Biennial Oscillation - QBO) na estratosfera e por uma oscilação semi-anual (Semi-Annual Oscillation SAO) acima de $35 \mathrm{~km}$ de altura (ANDREWS et al., 1987; HOLTON, 1992).

Acredita-se que a circulação zonal média, assim como as SAO's da média atmosfera equatorial e de baixas latitudes, sejam controladas principalmente por ondas atmosféricas que são geradas na baixa atmosfera e se propagam, sob condições favoráveis, horizontal e verticalmente, alcançando alturas próximas à mesopausa. A redução de momentum associada com a quebra ou a dissipação destas ondas, bem como mecanismos convectivos, desempenha um papel importante na manutenção da circulação atmosférica global na média atmosfera (YOSHIDA et al., 1999).

Estudos recentes mostram a presença da SAO na temperatura mesosférica para diversas latitudes, utilizando a técnica de fotometria, como por exemplo: Maui $\left(20,8^{\mathrm{o}} \mathrm{N}\right)$ (ZHAO et al., 2007); Cachoeira Paulista (22,7응 S) (TAKAHASHI et al., 1995) e Tirunelveli $\left(8,7^{\circ}\right.$ N) (SRIDHARAN et al., 1999). Também é possível encontrar registros na literatura da presença dessa oscilação quando a temperatura é medida por meio do instrumento TIMED/SABER (HUANG et al., 2006) e por outras técnicas, como o Wind Imager Interferometer a bordo do Upper Atmosphere Research Satellite, WINDII UARS (SHEPHERD et al., 2006).

Neste trabalho, são investigadas essas oscilações na temperatura a uma altitude de $\sim 90 \mathrm{~km}$ sobre São João do Cariri-PB ( $7,4^{\circ}$ S; 36,5 W). Para tanto, foi feita a aquisição da temperatura diária por meio do radar meteórico SKiYMET durante o período de julho de 2004 a junho de 2009. Adicionalmente, foram obtidos os dados de temperatura através do instrumento Sounding of the Atmosphere by Roadband Emission Radiometer (SABER), a bordo do satélite Thermosphere-Ionosphere-Mesosphere Energetics and Dynamics (TIMED) para os anos de 2005 a 2007 e ainda por fotometria do $\mathrm{OH}$ durante o período de 2004 a 2007.

\section{Materiais e métodos}

A determinação da temperatura da alta atmosfera é de extrema importância para realização de estudos sobre a dinâmica, a física e a química dessa região. Porém, a sua determinação in loco só é possível com o uso de foguetes, os quais permitem apenas realizar medidas por um curto intervalo de tempo, com alto custo por experimento. Várias técnicas 
são, portanto, utilizadas para determinar a temperatura numa faixa de altitudes. As técnicas óticas, passivas ou ativas, são as mais comumente empregadas para observar a temperatura da alta atmosfera e seus equipamentos podem ser instalados a bordo de satélites, aviões ou, mais usualmente, no solo. Nesta seção será descrito o princípio de funcionamento do radar meteórico SKiYMET, do fotômetro Multi-3 e do TIMED/SABER.

\section{RADAR METEÓRICO}

A pesquisa desenvolvida neste trabalho teve como base as observações da temperatura mesosférica na região próxima à mesopausa. Os dados de temperatura foram estimados a partir das informações registradas pelo radar meteórico do tipo SKiYMET que opera em São João do Cariri-PB $\left(7,4^{\circ} \mathrm{S} ; 36,5^{\circ} \mathrm{W}\right)$ no período compreendido de julho de 2004 a junho de 2009.

O radar meteórico é um instrumento utilizado para a observação de meteoros que entram na atmosfera terrestre frequentemente. Destas observações, podem ser medidos vários parâmetros de interesse atmosférico e astronômico, tais como: o fluxo de meteoros, o vento neutro e o coeficiente de difusão ambipolar. $O$ radar meteórico em estudo tem por função a detecção de meteoros e a medição de ventos, na faixa de altitudes de $78 \mathrm{~km}$ a 110 km (mesosfera e baixa termosfera).

Quando um meteoróide adentra na atmosfera, ele rapidamente é vaporizado, deixando um traço de gás ionizado em sua trajetória. Este traço pode ser detectado pelo radar meteórico, que transmite um curto pulso de energia na forma de ondas de rádio de uma antena em Very High Frequency (VHF). Parte desta energia é refletida pela trilha meteórica ionizada sendo detectada por um conjunto de antenas receptoras (HOCKING et al., 2001).

O radar meteórico do Observatório de Luminescência Atmosférica da Paraíba (OLAP), instalado em São João do Cariri-PB, detecta os traços de meteoros sobre todo o céu, e opera automaticamente durante 24 horas por dia detectando cerca de 3000 a 6000 meteoros úteis por dia. Tal radar opera em frequência de $35,24 \mathrm{MHz}$, uma taxa de repetição de pulsos de 2144 pps e um pico de potência de $12 \mathrm{~kW}$. Os cálculos realizados com os ecos detectados fornecem muitas informações sobre a natureza do meteoro e sua velocidade de entrada na atmosfera.

A detecção do meteoro é realizada através de dois processos sucessivos. Primeiro é feita a identificação do potencial do sinal, através de análise da fase e quadratura. No segundo estágio, esses sinais são detalhadamente examinados e confirmados como sinais meteóricos ou não. Determina-se, então, os ventos e temperaturas nas regiões cujas altitudes na atmosfera estão compreendidas entre 80 e $100 \mathrm{~km}$, utilizando as trilhas meteóricas deixadas por pequenos meteoróides ao entrarem na atmosfera.

Aqui o cálculo da temperatura mesosférica através do radar meteórico SKiYMET foi feito segundo os trabalhos de Hocking $(1999 ; 2004)$ em que é realizado o cálculo do gradiente de temperatura na altura onde ocorre a maior contagem de ecos meteóricos.

Para latitudes médias e altas, a mesopausa encontra-se acima do pico de contagem de meteoros durante a maior parte do ano, sendo o gradiente de temperatura bem definido nesta região. No entanto, no verão há ocasiões em que a mesopausa desce abaixo do pico de meteoros, o que implica em um gradiente de temperatura positivo. Usando resultados de estudos por meio de modelos e de dados do Lidar e de experimentos com foguetes, Hocking chegou à seguinte equação como estimativa do gradiente de temperatura como função da latitude e do tempo na altura de pico da contagem de meteoros: 


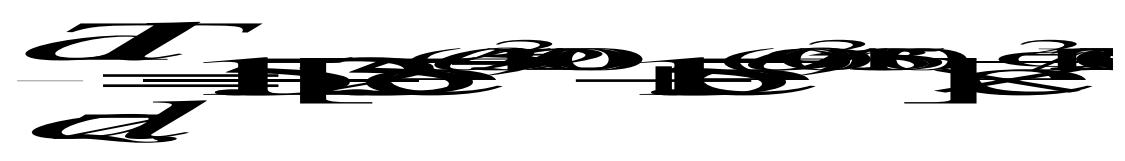

Sendo $\Phi$ o deslocamento temporal em número de dias da metade de junho no Hemisfério Norte ou deslocamento temporal em número de dias da metade de dezembro, no Hemisfério Sul. Posteriormente, Hocking (2004) melhorou esse modelo para o gradiente de temperatura, minimizando as incertezas envolvidas no cálculo de T.

\section{FOTÔMETRO MULTI-3}

O fotômetro é um equipamento destinado a medir a intensidade da radiação de um fluxo de fótons e, em alguns casos, através dessa medida, calcular a temperatura da região da qual provém a emissão. A medida da intensidade é feita através de comparações quantitativas por meio de dispositivos fotoelétricos e esse é um dos equipamentos mais utilizados no estudo da temperatura devido a sua fácil operação e manuseio, e também ao seu baixo custo. Nesse estudo é utilizado um fotômetro, denominado de Multi-3, que foi construído pelo INPE (Instituto Nacional de Pesquisas Espaciais) para medir a intensidade zenital do airglow das emissões do OI5577, OI6300, $\mathrm{NaD}, \mathrm{OH}(6,2)$ e O2(0,1), e ainda as temperaturas rotacionais do $\mathrm{OH}(6,2)$ e $\mathrm{O} 2(0,1)$. A temperatura da atmosfera em torno de 87 $\mathrm{km}$ de altitude pode ser estimada a partir do espectro de emissão de várias bandas vibrarotacionais do $\mathrm{OH}$. Na maioria dos casos, a determinação é feita a partir do espectro da banda $\mathrm{OH}$ (6-2), mais especificamente as emissões das linhas P1(2) e P1(4), ambas centradas em $8340 \AA ̊$ e $8466 \AA$, respectivamente. Aqui o que se faz é comparar as medidas feitas pelo fotômetro Multi-3 que se encontra instalado no solo com o espectro teórico da banda. Medindo-se a intensidade de pelo menos duas linhas rotacionais é possível inferir a temperatura rotacional da molécula a partir da razão entre as intensidades dessas linhas. A temperatura rotacional da molécula pode ser utilizada como uma estimativa da temperatura cinética da atmosfera na região da camada de emissão da hidroxila, pois o número de colisões dessa molécula, por unidade de tempo, com os constituintes majoritários na região de emissão é suficientemente maior do que o intervalo de tempo até o equilíbrio radiativo. Assim, supõe-se que as populações rotacionais podem atingir o equilíbrio térmico com o ambiente antes da emissão de fótons. Detalhes do cálculo do espectro teórico dessa banda e dos procedimentos experimentais empregados no cálculo da temperatura rotacional podem ser obtidos em diversos trabalhos (BURITI, 1997; BAGESTON, 2006).

$\mathrm{O}$ espectro do $\mathrm{OH}(6,2)$ é formado por várias emissões resultantes de transições vibra-rotacionais para estados de menor energia. A faixa de emissão desta banda, compreendendo as principais linhas (maior intensidade), vai de $8280 \AA$ até $8600 \AA$. A temperatura rotacional pode ser obtida a partir da razão entre as intensidades de duas emissões e pode ser interpretada como sendo a temperatura cinética; isto ocorre quando se supõe que os estados excitados obedecem a uma distribuição de Boltzmann e estão em equilíbrio térmico com a atmosfera (SUZUKI \& TOHMATSU, 1976; SHE \& LOWE, 1998).

\section{TIMED/SABER}

O SABER (Sounding of the Atmosphere using Broadband Emission Radiometry) é um instrumento destinado a medir vários parâmetros atmosféricos entre 10 e $180 \mathrm{~km}$ de altitude. Ele foi lançado em dezembro de 2001 a bordo do satélite TIMED (Thermosphere 
Ionosphere Mesosphere Energetics and Dynamics) da NASA (TIMED Web Site, 2010). O SABER utiliza um radiômetro multi-espectral para medir a radiação vinda do horizonte. $O$ perfil de temperatura da atmosfera entre 10 e $105 \mathrm{~km}$ com resolução de $2 \mathrm{~km}$ é obtido através da medida da intensidade da emissão do $\mathrm{CO}_{2}$ em $15 \mu \mathrm{m}$ e $4.3 \mu \mathrm{m}$, aplicando análise de transferência radiativa (MERTENS et al., 2004). Essas medidas são então combinadas para fornecer a temperatura cinética $\mathrm{Tk} / \mathrm{CO}_{2}$ simultaneamente.

O instrumento SABER é apenas um dos quatro instrumentos lançados a bordo do satélite TIMED e consiste de um radiômetro operando no infravermelho próximo, entre 1,27 e $17 \mu \mathrm{m}$ capaz de medir perfis verticais de emissões de vários gases da atmosfera terrestre. Através de uma visada de limbo o SABER é capaz de sondar continuamente a atmosfera terrestre durante o dia e a noite, permitindo assim o estudo da variabilidade diária dos principais parâmetros atmosféricos, dentro de uma cobertura global. O TIMED também tem fornecido uma cobertura geográfica sem precedentes para o estudo e determinação das ondas e marés na região MLT (Mesosfera e baixa Termosfera).

\section{Resultados}

Os períodos de aquisição dos dados de temperatura foram diferentes, devido a fatores diversos, tais como: noites de Lua Cheia, que impossibilitam as medições do fotômetro Multi-3, manutenção do sistema do radar meteórico SKiYMET; dentre outros, sendo, sempre que possível, a frequência de aquisição amostral diária. A Tabela 1 apresenta os períodos de aquisição de dados para cada um dos instrumentos.

Tabela 1: Período de observação para cada instrumento.

\begin{tabular}{|c|c|}
\hline INSTRUMENTO & PERÍODO DE OBSERVAÇÃO \\
\hline Radar Meteórico SKiYMET & Julho de 2004 - Junho de 2009 \\
\hline Temperatura rotacional & Janeiro de 2004 - Julho de 2007 \\
\hline TIMED/SABER & Janeiro de 2005 - Dezembro de 2007 \\
\hline
\end{tabular}

A Figura 1 mostra o comportamento da temperatura mesosférica obtida pelo radar meteórico no período a uma altitude aproximada de $90 \mathrm{~km}$. Em um intervalo de 1826 dias, foram obtidos 1712 dias de observações.

A temperatura é obtida na altitude onde ocorre a maior contagem de ecos de meteoros. Num dia, é usual registrar milhares de ecos a fim de inferir a temperatura de um único dia. Para o período de dados relativo ao radar meteórico SKiYMET instalado em São João do Cariri-PB, esta altitude média ficou em torno de 90,4 $\pm 0,45 \mathrm{~km}$ e a temperatura média foi de $226,2 \pm 13,8 \mathrm{~K}$. A razão para se ter apenas um valor da temperatura por dia é para garantir que o gradiente médio de temperatura esteja bem definido e assim, garantir uma maior precisão quando determinar a temperatura absoluta através da inclinação do gráfico do $\log (\mathrm{Da})$ em função da altitude de referência (HOCKING, 1999) já que não é utilizada informação de pressão determinada por modelos. Esta técnica, que supõe que a temperatura varia linearmente com a altitude, funciona muito bem quando a mesopausa está abaixo do pico de contagem de meteoros, pois isso mantém um gradiente de temperatura mais uniforme. Os valores relativamente baixos apresentados em algumas ocasiões, a priori, não podem ser atribuídos ao número de pontos (ecos de meteoros) utilizados para o cálculo da temperatura visto que, em média, mais de 3000 mil pontos são utilizados para este fim. Para o período de observação deste trabalho, o número médio de ecos de meteoros detectados ficou em torno de 4192 por dia. 


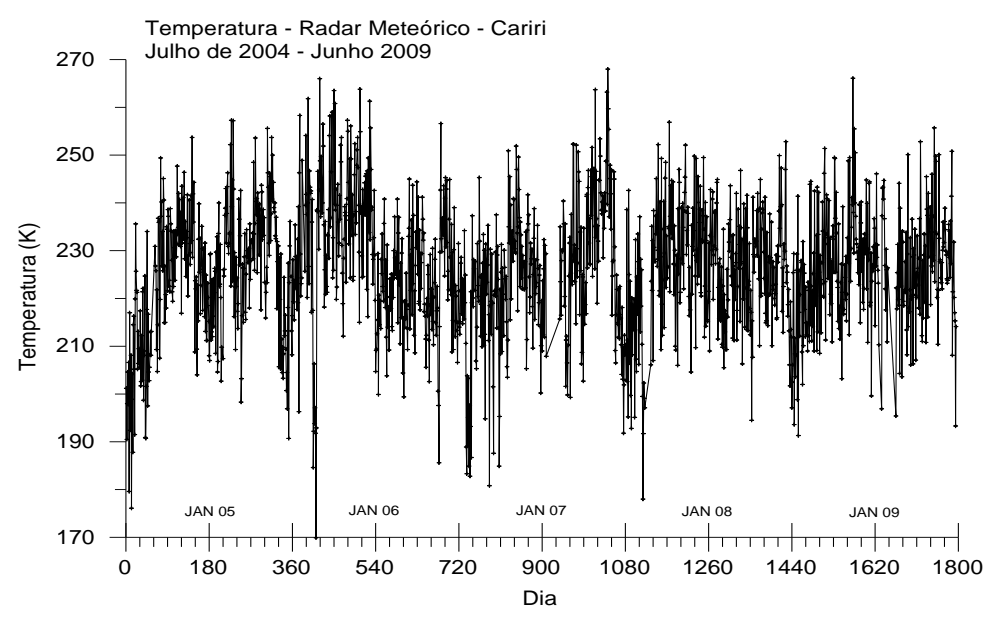

Figura 1: Gráfico da temperatura mesosférica medida pelo radar meteórico SKiYMET para o período de julho de 2004 a junho de 2009 em São João do Cariri- PB.

Observa-se que existe um comportamento oscilatório bastante pronunciado para os dados do radar meteórico SKiYMET, mas também é possível detectar uma variabilidade periódica igualmente significativa. Para observar de maneira mais clara o comportamento desses dados, foi feita uma média anual dos dados de temperatura medidos por esse instrumento, de modo que o conjunto de cinco anos de dados resultou em um só (Figura 2), ou seja, com apenas um valor de temperatura para cada dia do ano.

Analisando os dados do fotômetro Multi-3, percebe-se que o comportamento oscilatório se repete ao longo da série de dados. Os anos de 2004, 2005 e 2006 foram anos de pluviosidade intensa na região do Cariri Paraibano, consequentemente esses anos apresentaram muitas noites de céu encoberto, por isso os dados medidos usando essa técnica foram bastante escassos, devido à necessidade de condições especiais de observação. A Figura 3 mostra o gráfico da temperatura rotacional do $\mathrm{OH}$ na mesosfera para o período de janeiro de 2004 a julho de 2007. Observa-se um comportamento oscilatório semelhante àquele mostrado pelos dados do radar meteórico SKiYMET, embora aqui os dados sejam mais esparsos, pois no intervalo de janeiro de 2004 a julho de 2007 tivemos apenas 274 dias de observações. A temperatura rotacional média foi de $203,83 \pm 9,0 \mathrm{~K}$.

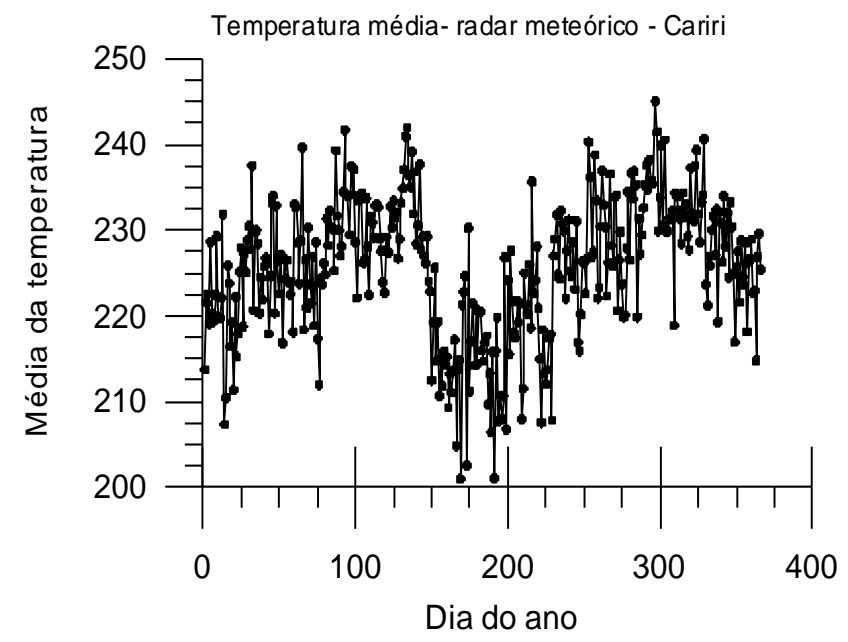

Figura 2. Gráfico da média anual da temperatura mesosférica medida pelo radar meteórico SKiYMET para os anos de 2004 a 2009 em São João do Cariri -PB. 


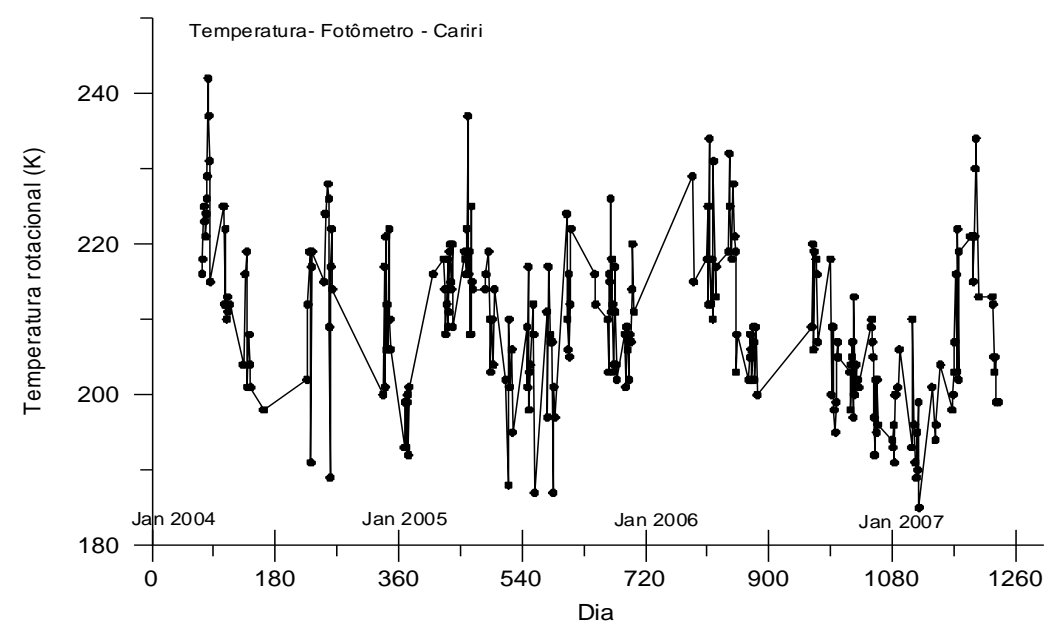

Figura 3. Gráfico da temperatura rotacional do $\mathrm{OH}$ mesosférico medida pelo fotômetro Multi-3 durante o período de janeiro de 2004 a julho de 2007 em São João do Cariri - PB.

Os dados de temperatura medida pelo instrumento TIMED/SABER estão plotados na Figura 4. Tais dados foram tomados para as altitudes de 85, 90 e $95 \mathrm{~km}$ de altitude. Para $85 \mathrm{~km}$ de altitude, a temperatura média observada ficou em torno de $197,9 \pm 9 \mathrm{~K}$, para $90 \mathrm{~km}$ o valor médio ficou em torno de 192,1 $\pm 9,9 \mathrm{~K}$ e para $95 \mathrm{~km}$ de altitude a temperatura média foi de $\sim 180,9 \pm 11,3 \mathrm{~K}$.

Comparando esses dados com aqueles do fotômetro Multi-3 e do radar meteórico SKiYMET percebe-se que a temperatura medida pelo TIMED/SABER é razoavelmente menor que aquelas apresentadas pelos outros instrumentos para a latitude em estudo.

Conhecido o comportamento da temperatura quando medida por cada instrumento, o objetivo agora é fazer uma análise espectral da temperatura para identificar os tipos de variações existentes. A metodologia para realização deste estudo foi basicamente a aplicação do método de Lomb-Scargle (LS) para determinação de possíveis oscilações nas séries temporais (LOMB, 1976; SCARGLE, 1982) e, em seguida, determinação das fases e amplitudes através da aplicação de método de análise harmônica, mais especificamente pelo método de mínimos quadrados. Ao utilizar o método LS foi obtido o espectro da temperatura. Os maiores picos são os harmônicos mais relevantes e somente eles devem ser utilizados para a reconstrução da curva.

Portanto, as séries temporais foram submetidas à analise de LS, em que foram identificadas as oscilações mais relevantes para a temperatura proveniente de cada um dos instrumentos. Procede-se então ao teste de Horne e Baluinas (1986), e em seguida, utilizou-se a análise harmônica e foi feita uma série de ajustes ou "fits" para encontrar as amplitudes e fases das oscilações mais significativas. Para o conjunto de dados coletados pelo radar meteórico SKiYMET, tem-se o periodograma do LS dado na Figura 5. Pode-se detectar as SAO e AO, que são bastante acentuadas, o que é esperado. Além dessas, temos a oscilação de 270 dias e a oscilação quase bi-anual, QBO. Foi feito o teste de Horne e Baluinas (1986) para verificar se a oscilação de $\sim 270$ dias é resultante de um vazamento espectral, ou seja, se ela foi gerada pelo 
próprio instrumento, mas o resultado mostra que essa oscilação está realmente presente nos dados do radar meteórico SKiYMET.

A Tabela 2 mostra as amplitudes e fases das oscilações mais significativas, com seus respectivos desvios padrão. Observa-se que a variação semianual tem a maior amplitude, o que mostra uma clara predominância da SAO no período estudado. A Figura 6 apresenta o ajuste feito pelo método dos mínimos quadrados usando os harmônicos da Tabela 2 e a comparação com a média corrida da temperatura para 21 dias.
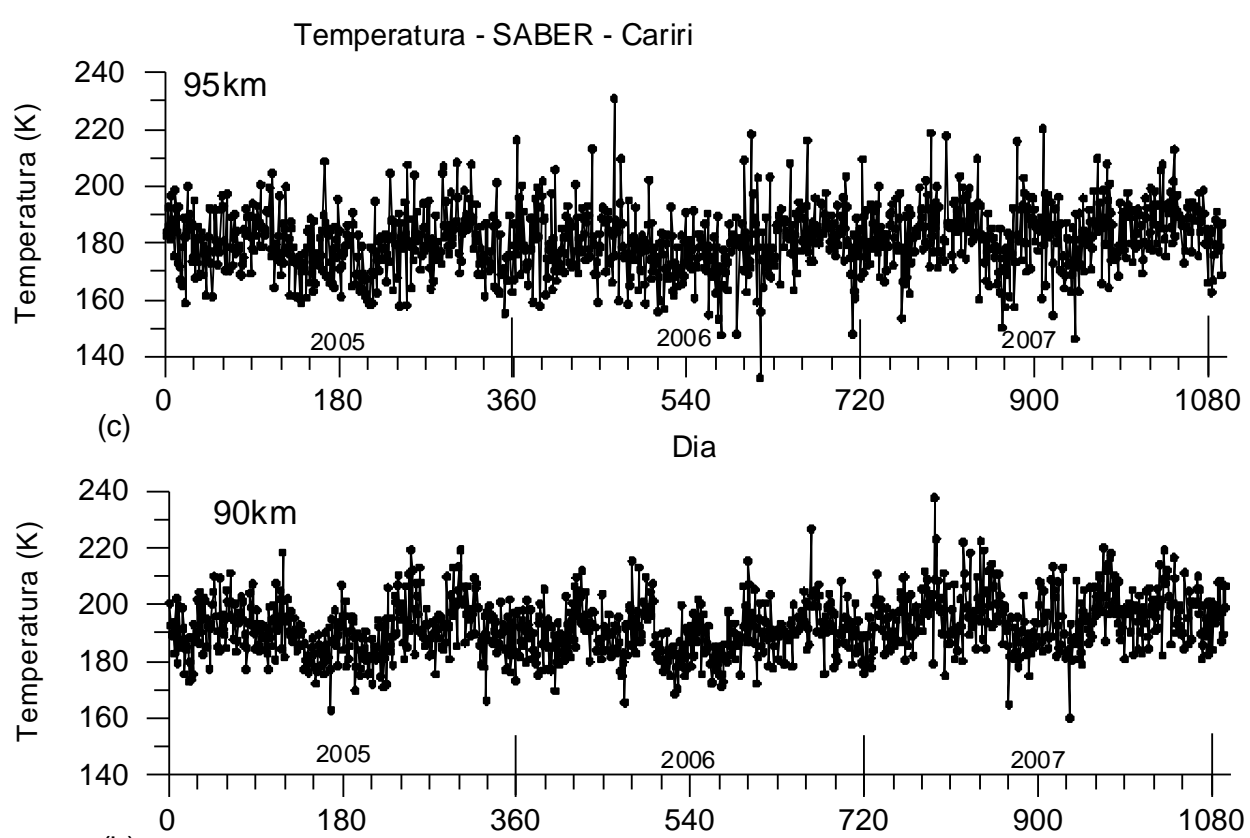

(b)

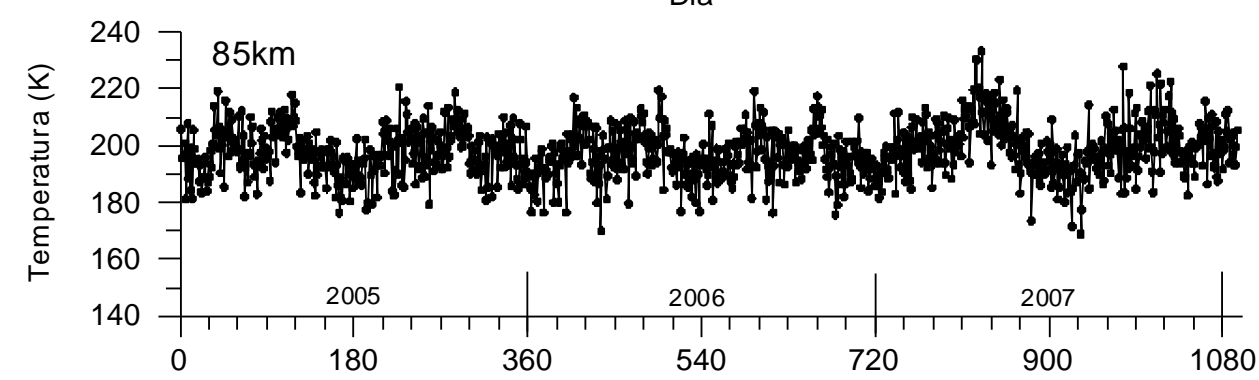

(a)

Dia

Figura 4: Gráfico da temperatura mesosférica medida pelo TIMED/SABER a uma altitude de (a) 85, (b) 90 e (c) $95 \mathrm{~km}$ para o período de janeiro de 2005 a dezembro de 2007 em São João do Cariri - PB. 


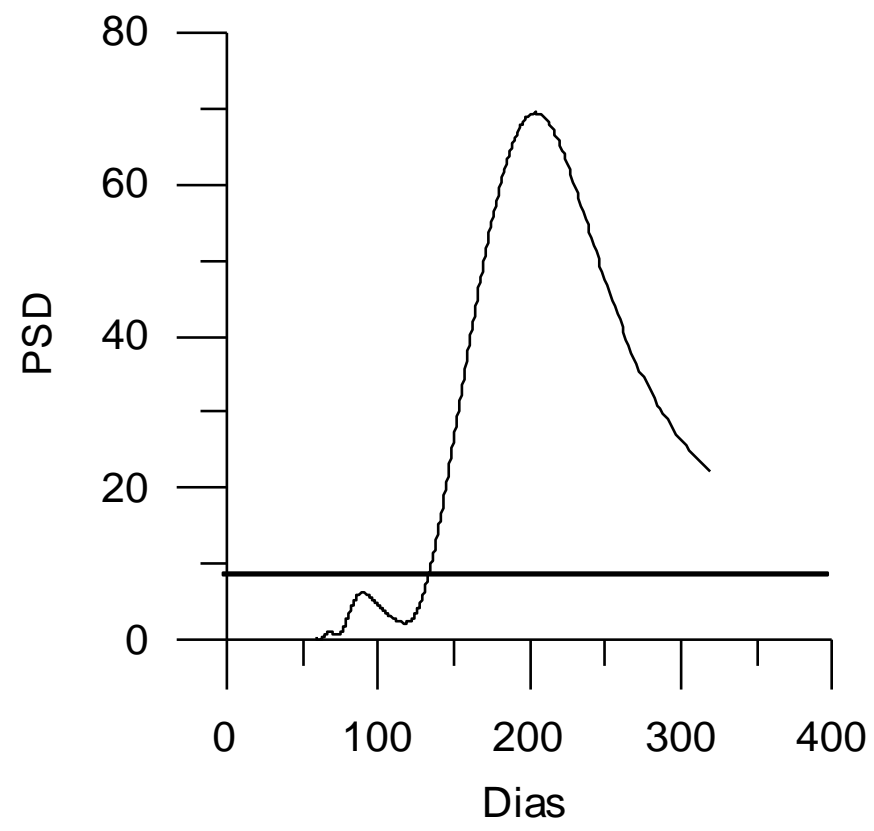

Figura 5: Periodograma (Power Spectral Density - PSD) para temperatura obtida pelo radar meteórico SKiYMET no período de julho de 2004 a junho de 2009. A linha horizontal representa o nível de confiança (95\%).

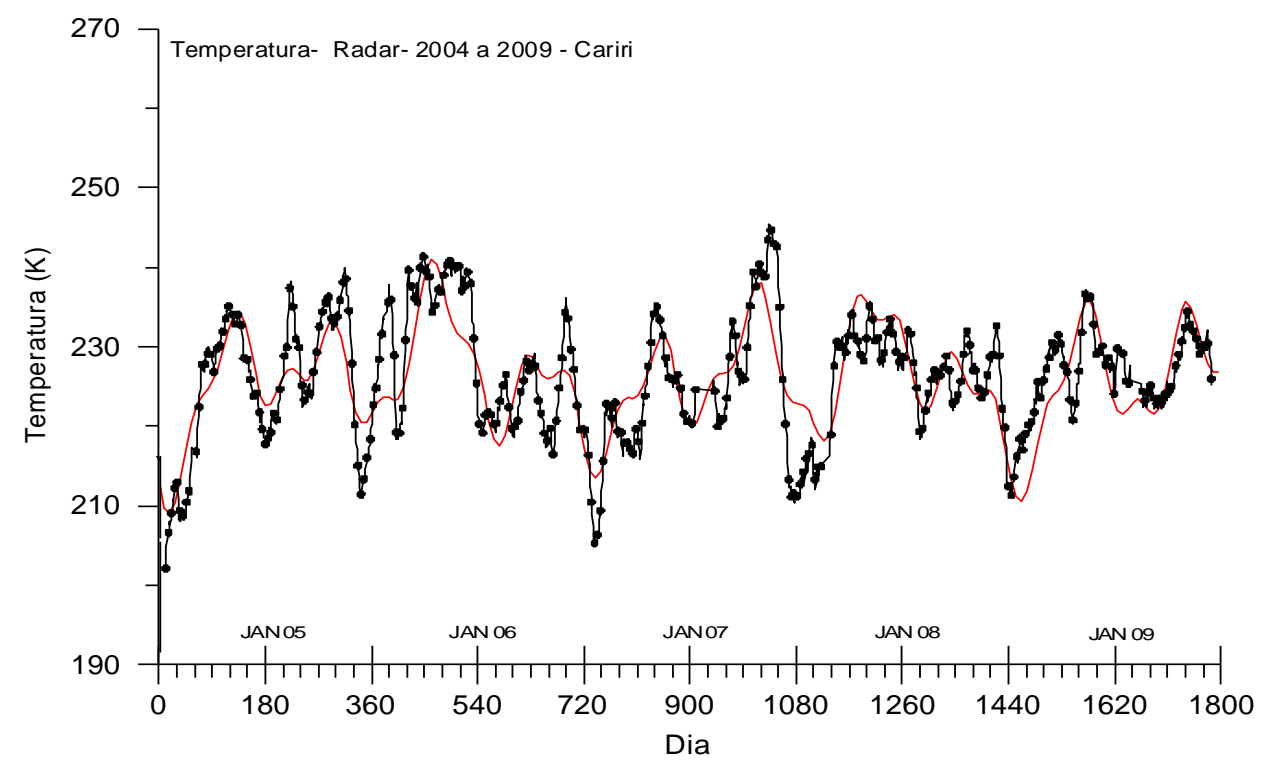

Figura 6: Comparação entre o ajuste feito pelo método dos mínimos quadrados (linha vermelha) e a média corrida de 21 dias da temperatura medida pelo radar meteórico SKiYMET instalado em São João do Cariri-PB no período de julho de 2004 a junho de 2009. 


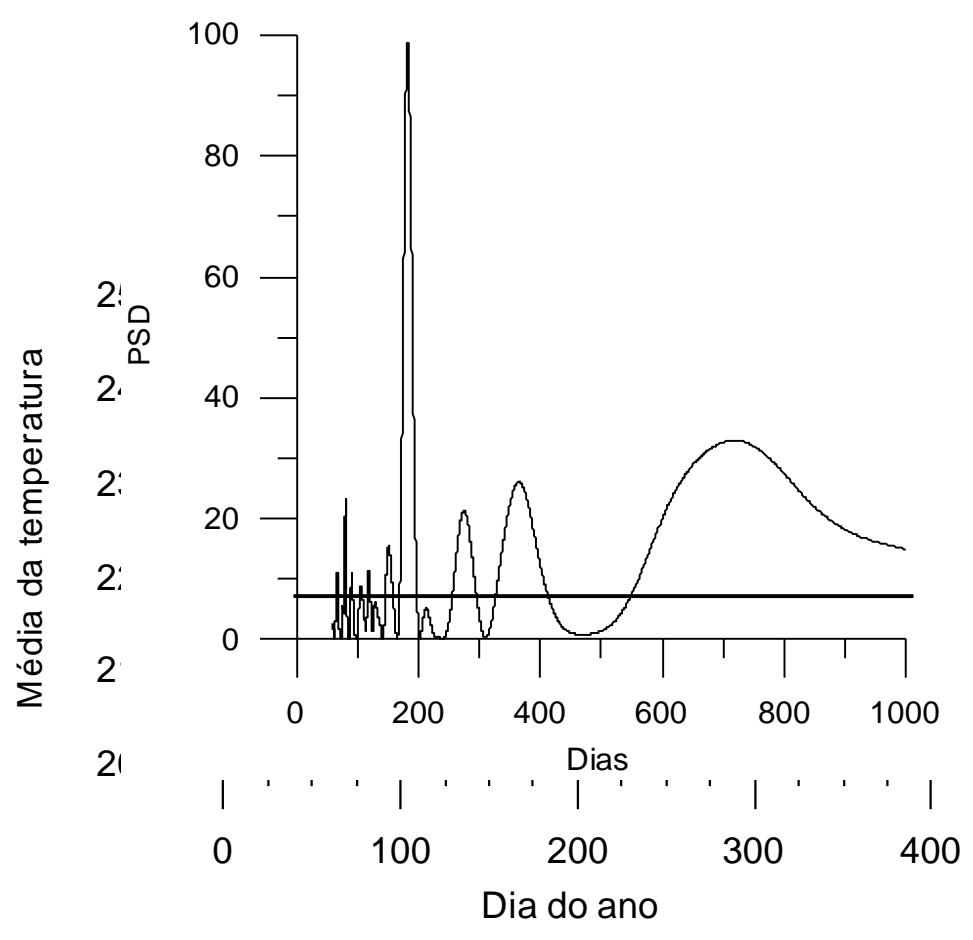

Figura 7: Comparação entre o ajuste feito pelo método dos mínimos quadrados (linha vermelha) e a média diária anual da temperatura medida pelo radar meteórico SKiYMET, no período de observação, evidenciando a SAO.

Tabela 2: Amplitudes e fases das oscilações observadas na temperatura medida pelo radar meteórico SKiYMET em São João do Cariri- PB.

\begin{tabular}{|c|c|c|c|c|}
\hline HARMÔNICO & AMPLITUDE (K) & DESVIO & FASE (Dias) & DESVIO \\
\hline 80 & 2,8 & 0,4 & 57,8 & 1,9 \\
\hline 181,7 & 6,2 & 0,4 & 109,5 & 1,9 \\
\hline 274,5 & 2,4 & 0,4 & 173,9 & 7,7 \\
\hline 364,5 & 3,0 & 0,4 & 195,7 & 8,3 \\
\hline 718 & 3,4 & 0,4 & 401,3 & 14,2 \\
\hline
\end{tabular}

Figura 8: Periodograma (Power Spectral Density - PSD) para temperatura média obtida pelo radar meteórico SKiYMET no período de observação. A linha horizontal representa o nível de confiança (95\%).

Na Figura 7 é feita a comparação entre a média diária da temperatura medida pelo radar meteórico SKiYMET e um ajuste das oscilações SAO e AO. Pode-se observar que a média anual da temperatura apresenta uma dupla corcova, ou duplos picos, em torno dos equinócios, o que sinaliza a presença de uma SAO. Desta forma, o ajuste realizado na média e plotado na Figura 8 foi feito com o objetivo de verificar a presença deste sinal na temperatura média. Conforme relatado por Zhao (2007), a curva com a forma senoidal e máximos em torno 
dos equinócios é uma clara evidência do sinal semi-anual e o pico mais acentuado em torno do equinócio de outono (UT day 110) demonstra a existência de um sinal anual adicional, ainda que mais tênue que o primeiro.

Observou-se que as oscilações menores, presentes na média diária anual para os dados do radar meteórico SKiYMET, são filtradas, restando apenas a de maior amplitude, neste caso a SAO. A amplitude e fase desta oscilação foram de 7,56K e 93,8 dias, respectivamente.

O periodograma de LS para o conjunto de dados coletados pelo fotômetro Multi-3 não será exibido aqui por questão de concisão. $\mathrm{O}$ mesmo evidenciou as oscilações mais significativas observadas, que foram a de 120 dias, a $\mathrm{SAO}$, a $\mathrm{AO}$ e a $\mathrm{QBO}$, essa última com uma amplitude razoavelmente menor que aquela apresentada pelos dados do radar meteórico SKiYMET. Neste caso, a QBO e a AO não são muito confiáveis devido aos dados do fotômetro Multi-3 terem sido muito esparsos. Após o teste de Horne e Baluinas (1986), a oscilação de 120 dias não persiste, uma vez que sua amplitude diminui bastante, ficando abaixo do nível de significância de 95\%, o que mostra que essa pode ser uma oscilação fictícia, provocada por flutuações nos dados, o que é chamado às vezes de oscilação produto do vazamento espectral. Mais uma vez a presença da SAO é incontestável.

Para a temperatura medida pelo TIMED/SABER tomou-se três periodogramas, um para cada faixa de altitude, que também não serão apresentados aqui. Em $85 \mathrm{~km}$ observase uma SAO bastante pronunciada, enquanto que não há presença das oscilações QBO e AO, mas surge aqui um harmônico de $\sim 90$ dias. Todas essas oscilações foram consideradas dentro dos limites de significância estatística da análise harmônica pelo teste de Horne e Baluinas (1986).

O ajuste pelo método do LS para os dados do TIMED/SABER a uma altitude de 90 $\mathrm{km}$, além da SAO e AO, mostra que novamente surge o harmônico de 90 dias e um outro de $\sim 240$ dias, que também foi detectado pelos dados a $85 \mathrm{~km}$ de altura. Por sua vez, o periodograma para os dados do TIMED/SABER a $95 \mathrm{~km}$ de altitude mostra oscilações mais acentuadas são novamente a $\mathrm{SAO}$, a $\mathrm{AO}$ e a de 90 dias. Chama-se a atenção para o fato de que esta última apareceu para todos os dados do TIMED/SABER, ou seja, para as três faixas de altitude estudadas por meio desse instrumento, e ainda, para os dados do radar meteórico SKiYMET. Aqui também o teste de Horne e Baluinas revelou que as oscilações de fato estão presentes na série de dados.

Para os dados do TIMED/SABER e do fotômetro Multi-3 também se pode afirmar que o ajuste do LS foi satisfatório. $O$ uso da média corrida de 35 dias para os dados do TIMED/SABER foi feito devido ao grande número de pontos que é disponibilizado por esse instrumento.

\section{Conclusões}

Este estudo estabeleceu a presença de uma $\mathrm{AO}$, bem como de uma SAO nas temperaturas mesosféricas medidas pelo radar meteórico SKiYMET para uma baixa latitude no Hemisfério Sul $\left(7,4^{\circ} \mathrm{S}\right)$. Como foi mostrado, o espectro referente à temperatura na mesosfera medida por esse instrumento no período compreendido entre julho de 2004 a junho de 2009 apresenta energia espectral para a banda periódica de $\sim 182$ dias durante o intervalo, com 
amplitude de $\sim 6,2 \mathrm{~K}$. O espectro apresenta ainda energia espectral para banda periódica em torno de $\sim 365$ dias e $\sim 720$ dias e episódios de energia espectral fraca na banda periódica de $\sim 90$ e $\sim 270$ dias.

Com respeito aos dados obtidos pelo fotômetro Multi-3, surgiu também a evidência de uma forte $\mathrm{SAO}$ e de $\mathrm{AO}$ na temperatura rotacional do $\mathrm{OH}$ entre janeiro de $2004 \mathrm{e}$ julho de 2007. A presença da QBO não ficou clara, devido, principalmente, ao pequeno número de pontos coletados.

A temperatura medida pelo TIMED/SABER entre janeiro de 2005 e dezembro de 2007 naturalmente apresentou variações conforme a altitude. Para $85 \mathrm{~km}$ de altitude, foi detectada uma SÃO, uma oscilação de $~ 90$ e ainda uma fraca oscilação adicional de 247 dias. No nível de $90 \mathrm{~km}$ as oscilações predominantes foram a SÃO, AO, oscilação de 90 dias e uma fraca oscilação de $\sim 240$ dias. Finalmente, no nível de $95 \mathrm{~km}$, as principais oscilações encontradas foram a SAO, a AO, e a oscilação de $\sim 90$ dias.

As oscilações do tipo SAO e AO, 182 e 365 dias, respectivamente, são bem conhecidas. A SAO foi relatada por Takahashi et al. (1995) usando a fotometria do OH para duas localidades, Fortaleza $\left(3,9^{\circ} \mathrm{S}\right)$ e Cachoeira Paulista $\left(22,7^{\circ} \mathrm{S}\right)$, com amplitudes distintas de $\sim 8,5 \mathrm{~K}$ (Fortaleza) e $\sim 3,5 \mathrm{~K}$ (Cachoeira Paulista). Zhao et al. (2007) também detectaram essas oscilações em dados de temperatura do instrumento MTM (Mesospheric Temperature Mapper), da Universidade de Utah, na região de Maui $\left(20,8^{\circ} \mathrm{N}\right)$. Eles mediram uma SAO com amplitude de $\sim 3,8 \mathrm{~K}$ e uma AO de amplitude $\sim 1,9 \mathrm{~K}$.

A QBO presente nos dados do radar meteórico SKiYMET também está bem caracterizada e é possível encontrar muitos registros da mesma na literatura. Huang et al. (2006) mostraram a existência dessa oscilação em dados do TIMED/SABER para o período entre 2002 e 2004 nas latitudes entre $48^{\circ} \mathrm{S}$ e $48^{\circ} \mathrm{N}$, e ainda uma SAO com amplitude bem pronunciada de $\sim 9 \mathrm{~K}$ para latitudes equatoriais. Segundo esses autores, as variações da SAO e da QBO na estratosfera e mesosfera são produzidas principalmente por processos dinâmicos associados com a circulação zonal que domina nessas camadas e que está confinada às latitudes equatoriais. A situação dinâmica que controla as variações de temperatura neste caso é semelhante àquela que controla a AO na mesosfera em altas latitudes (LINDZEN, 1981).

Nos dados do radar meteórico SKiYMET e do TIMED/SABER foi detectada uma oscilação de $\sim 9$ meses (250 a 270 dias), que não foi encontrada na literatura, mas que, no entanto, pode estar presente na temperatura como uma oscilação verdadeira ou como tendo sido gerada pelo próprio instrumento. Essa oscilação requer um estudo mais detalhado. Ainda se constatou que as medidas de temperatura feitas pelo TIMED/SABER estão razoavelmente abaixo daquelas feitas pelos demais instrumentos. Na Tabela 3 encontra-se um sumário dos resultados encontrados nesta pesquisa, note-se que as temperaturas médias foram tomadas sem o seu desvio padrão. Apenas a $\mathrm{SAO}$ e a $\mathrm{AO}$ foram observadas nos dados dos três instrumentos.

Observou-se que a temperatura medida pelo radar meteórico SKiYMET apresentou um valor médio (226,2 K) maior que a dos outros dois meios utilizados, sendo a temperatura medida pelo TIMED/SABER $(197,9 ; 192,1$ e 180,9 K para 85, 90 e $95 \mathrm{~km}$ de altitude, respectivamente), a que apresentou o menor valor médio na latitude de observação. Esse valor ficou em torno de 203,83 K para o fotômetro Multi-3.

Não é possível afirmar que o instrumento TIMED/SABER realmente tenha apresentado uma temperatura média menor que os demais devido à diferença de altitude em 
que esta foi coletada, ou se houveram falhas na detecção da temperatura por meio do radar meteórico SKiYMET durante o intervalo em que os dados foram medidos, de modo a fornecer uma temperatura média muito elevada. Para estudos posteriores sugere-se a análise da calibração desse instrumento. Entretanto, confirma-se a presença da SAO e da AO na temperatura da mesosfera equatorial.

Tabela 3 : Sumário dos resultados obtidos neste trabalho.

\begin{tabular}{|c|c|c|c|}
\hline INSTRUMENTO & MÉDIA DE T (K) & PRINCIPAIS OSCILAÇÕES & ALTITUDE \\
\hline $\begin{array}{c}\text { Radar Meteórico } \\
\text { SKiYMET }\end{array}$ & 226,2 & SAO, AO, QBO, 80 e $\sim 270$ dias & $90 \mathrm{~km}$ \\
\hline Fotômetro Multi-3 & 203,83 & SAO & $87 \mathrm{~km}$ \\
\hline TIMED/SABER & 197,$9 ; 192,1$ e 180,9 & SAO, AO, $\sim 90$ e $\sim 250$ dias & 85,90 e $95 \mathrm{~km}$ \\
\hline
\end{tabular}

\section{Referências Bibliográficas}

ALVES, E. O., Identificação de Ondas Atmosféricas Ultra-Rápidas de Kelvin nos Ventos Meteóricos Sobre São João do Cariri-PB $\left(7,4^{\circ}{ }^{\circ} ; 36,5^{\circ} \mathrm{O}\right)$, Dissertação de Mestrado em Meteorologia, Universidade Federal de Campina Grande, Campina GrandePB, 2007.

ANDREWS, D. G.; HOLTON, J. R.; LEOVY, C. B. Middle Atmosphere Dynamics. Orlando: Academic press, 1987. v. 489p.

BAGESTON, J. V. Determinação da temperatura da mesosfera superior utilizando um espectro-imageador. 152p. Dissertação de Mestrado em Geofísica Espacial, Instituto Nacional de Pesquisas Espaciais, São José dos Campos-SP, 2006.

BURITI, R. A. Estudo de parâmetros de ondas de gravidade por medidas simultâneas de radar MU e fotômetro em Shigaraki (35N, 136ㅌ) Japão. 231p. Tese de Doutorado em Geofísica Espacial, Instituto Nacional de Pesquisas Espaciais, São José dos Campos-SP, 1997.

HOCKING, W. K., Temperatures using radar meteor decay times. Geophysical Research Letters, v.26, n.21, p.3297-3300, 1999.

HOCKING, W. K., FULLER, B., VANDEPEER, B., Real-time determination of meteorrelated parameters utilizing modern digital technology. Journal of Atmospheric and SolarTerrestrial Physics, v. 63, p. 155-169, 2001.

HOCKING, W. K., Radar meteor decay rate variability and atmospheric 
consequences. Annales Geophysicae, v.22, p.3805-3814, 2004.

HORNE, J. H.; BALIUNAS, S. L. A prescription for period analysis of unevenly sampled time series. Astrophysical Journal, Part 1, v. 302, n. 2, p. 757-763, 1986.

HUANG, F. T., MAYR, H. G., REBER, C. A., RUSSELL, J. M., MLYNCZAK, M., MENGEL, J. G., Stratospheric and mesospheric temperature variations for the quasibiennial and semiannual (QBO and SAO) oscillations based on measurements from SABER (TIMED) and MLS (UARS), Annales Geophysicae, v. 24, p. 2131-2149, 2006.

LINDZEN, R. S., HOLTON, J.R., A theory of the quasi-biennial oscillation, Journal of the Atmospheric Science, v.25, p. 1095-1107, 1968.

LOMB, N. R. Least-squares frequency analysis of unequally spaced data. Astrophysics and Space Science, v. 39, n. 2, p. 447-462, 1976.

MERTENS, C. J., SCHMIDLIN, F. J., GOLDBERG, R. A., REMSBERG, E. E., PESNELL. W.D., RUSSELL, J. M., MLYNCZAK, M. G., LÓPEZ-PUERTAS, M., WINTERSTEINER, P. P., PICARD, R. H.,WINICK, J. R.,GORDLEY, L. L., SABER observations of mesospheric temperatures and comparisons with falling sphere measurements taken during the 2002 summer MaCWAVE campaign, Geophysical Research Letters, v.31, p. 3105-3110 , 2004.

SCARGLE, J. D. Studies in astronomical time series analysis. II - Statistical aspects of spectral analysis of unevenly spaced data. Astrophysics Journal, Part 1, v. 263, p. 835-853, 1982.

SHE, C. Y., LOWE, R. P. Seasonal temperature variations in the mesopause region at midlatitude comparison of lidar and hydroxyl rotational temperatures using WINDII:UARS OH Height profiles. Journal of Atmospheric and Solar-Terrestrial Physics, v.60, p.1573-1583, 1998.

SHEPHERD, M. G., LIU, G., SHEPHERD, G. G., Mesospheric semiannual oscillation in temperature and nightglow emission, Journal of Atmospheric and Solar-Terrestrial Physics, v. 68, p. 379-389, 2006.

SRIDHARAN, R., TAORI, A., GURUBARAN, S. , RAJARAM, R., SHEPHERD, M. G., First results on daytime mesopause $\mathrm{OH}$ rotational temperatures using ground-based photometry from equatorial latitudes, Journal of Atmospheric and Solar-Terrestrial Physics, v.61, p. 1131-1142, 1999.

SUZUKI, K., TOHMATSU, T. An interpretation of the rotational temperature of the 
airglow hydroxyl emissions. Planetary and Space Science, v.24, p. 665-671, 1976.

TAKAHASHI, H., CLEMESHA, B. R., BATISTA, P. P. , Predominant semi-annual oscillation of the upper mesospheric airglow intensities and temperatures in the equatorial region, Journal of Atmospheric and Terrestrial Physics, V.. 57, n. 4, p. 407-414, 1995.

TIMED WEB SITE. Timed- A mission to explore one of the last frontiers in Earth's atmosphere. Washington: NASA. Disponível em: <http://www.timed.jhuapl.edu> Acesso em 11 de outubro de 2010.

ZHAO, Y., TAYLOR, M. J., LIU, H. L, ROBLE , R. G. Seasonal oscillations in mesospheric temperatures at low-latitudes, Journal of Atmospheric and Solar-Terrestrial Physics, v.69, p. 2367-2378, 2007.

YOSHIDA, S., TSUDA, T., SHIMIZU, A., NAKAMURA, T., Seasonal variations of 3.0 3.8-day ultra-fast Kelvin waves observed with a meteor wind radar and radiosonde in Indonesia, Earth, Planets and Space, v. 51, p. 675-684, 2006. 